\title{
In Vivo FF-SS-OCT Optical Imaging of Physiological Responses to Photostimulation of Human Photoreceptor Cells
}

\author{
Dierck Hillmann, Clara Pfäffle, Hendrik Spahr, \\ Helge Sudkamp, Gesa Franke, \\ and Gereon Hüttmann
}

\subsection{Introduction}

The human vision system achieves an extraordinary level of performance, which is only recently matched by technical systems. Three components contribute to our vision capabilities: the optics of the eye, the light detection by the photoreceptor cells (PRCs), and the processing of the visual information in the neuronal structures of the retina and the brain. Diseases are known to interfere with physiological function of the vision system on all three levels: They may result in failing to

D. Hillmann

Thorlabs GmbH, Lübeck, Germany

Institute of Biomedical Optics, University of Lübeck, Lübeck, Germany

C. Pfäffle $\cdot$ H. Spahr · G. Franke

Institute of Biomedical Optics, University of Lübeck, Lübeck, Germany

H. Sudkamp

Institute of Biomedical Optics, University of Lübeck, Lübeck, Germany

Medical Laser Center Lübeck GmbH,

Lübeck, Germany

G. Hüttmann $(\bowtie)$

Institute of Biomedical Optics, University of Lübeck, Lübeck, Germany

Medical Laser Center Lübeck GmbH, Lübeck, Germany

Airway Research Center North (ARCN), Member of the German Center of Lung Research (DZL), Gießen, Germany form a high-quality image on the retina, loss of the photoreceptor cell function, or deficiencies in the neuronal processing of the visual information. Consequently, testing vision on all three levels is important for the diagnosis and therapy of ophthalmic diseases.

Subjective methods, which require patient feedback, and objective methods are available. Subjective techniques use the perception of the patient evoked by an optical stimulus to test the function of the visual system. They have the drawback to measure only the integral performance of all steps in vision and need cooperation of the patient. Subjective vision testing ranges from simple vision tests to perceptive investigations after stimulating single photoreceptor cells using adaptive optics [1, 2]. However, quantification is challenging since data are inherently based on subjective observations.

Objective methods measure visual function directly and give quantitative results on certain functional parameters, such as refraction, transparency of the optical media, field of view, or light sensitivity. Furthermore, objectively measuring visual function may often be the only option for children, elderly people, and other non-cooperating patients. A number of devices are currently used for healthy and pathological eyes, which include autorefractors, wavefront sensors, slit lamps, scanning laser ophthalmoscopes, and optical coherence tomography (OCT) devices. The state-of-the-art clinical method for an objective measurement of retinal 
function is electroretinography (ERG) [3]. ERG measures electrical potential changes of photoreceptor cells, inner retinal cells, and ganglion cells in response to specific stimuli. Multifocal ERG even provides spatial resolution, but the weak ERG signal does not allow testing of single cells.

However, there is currently no objective method to measure the function of individual photoreceptor cells, neurons, or their interaction. These measurements require sensitivity to the biochemical processes of vision and its neuronal processing; it also needs to be combined with cellular spatial resolution - a combination that is hardly achieved for human imaging today. Non-optical imaging by computer tomography (CT), ultrasound (US), or magnetic resonance imaging (MRI) does not provide the necessary spatial resolution for photoreceptor cells and neurons. Optical imaging may have the necessary temporal (millisecond) and spatial ( $2 \mu \mathrm{m}$ at full pupil size) resolution but lacks contrast for the relevant biochemical processes. Although ion and voltage sensitive fluorescent dyes [4] are available to study cellular function in cells, organ cultures, or animals, these dyes are not applicable since they are not approved for human use. And even if they were, the axial resolution of fluorescence imaging is not sufficient to separate the different layers of the human retina. Only the visual cycle itself, i.e., the change of opsins during optical stimulation, has intrinsic spectroscopic contrast and can be studied with high spatial resolution by densitometry [5] and two-photon excited autofluorescence [6]. Both methods give molecular information on the vision cycle and the regeneration of the retinal photopigment.

Changes of the photopigments are measurable since their interaction with light is intrinsic to their function. But the following steps of the photo-transduction cycle are biological processes that involve only changes of the concentration of optically non-active biomolecules and ions, as well as changes of the electrical potentials. In vivo, these changes are not directly measurable by optical means. However, secondary effects like changes of the refractive index, scattering, birefringence, or morphology [7, 8] have been suggested for a non-invasive measurement. Optical changes of the tissue that are correlated with retinal activity are called intrinsic optical signals (IOS). Despite their unspecific origin and small signal level, they allow non-invasive optical measurements of the activity of photoreceptor and neuronal activity [8,9]. Considerable efforts were invested since the end of the seventies to detect IOS [7, 8, 10]. Changes of scattering, reflection, or birefringence and also geometrical changes of photoreceptor and neuronal cells were detected ex vivo and in animals.

However, transferring these results to imaging of the retina in humans faced considerable problems. Ocular aberrations and the restricted numerical aperture confine imaging resolution. The sensitivity to changes of the optical tissue properties is limited by shot noise of the photons. Increasing irradiation levels on the retina improves the signal-to-noise ratio but is limited by the permissible irradiance of the fundus. Ocular motion causes blur and other artifacts in the images. Still, IOS were measured in vivo using fundus photography, laser scanning ophthalmoscopy (SLO) [11], and optical coherence tomography (OCT) [12]. The observed signals were dominated by noise since, as the vision process is associated with only small changes in scattering of membranes and cell organelles. Hence the observed intrinsic signals were of low quality, corrupted by motion artifacts [13], difficult to interpret and their physical origin was unclear. First hints, that phase sensitive imaging might be the key to reliably detecting IOS, were found by Jonnal et al. [14]. Using a high-speed flood illumination retina camera equipped with adaptive optics (AO), fluctuations of the reflections of cones were observed under stimulation. These fluctuations were explained by interference of the reflections of both ends of the outer segment, which is very sensitive to changes of the length or the refractive index of the outer segments. A quantiative analysis of these interferences is possible by OCT.

\subsection{Holographic Optical Coherence Tomography}

Fourier domain OCT (FD-OCT) is the state-ofthe-art technique for imaging the human retina. It obtains detailed cross-sectional and three- 

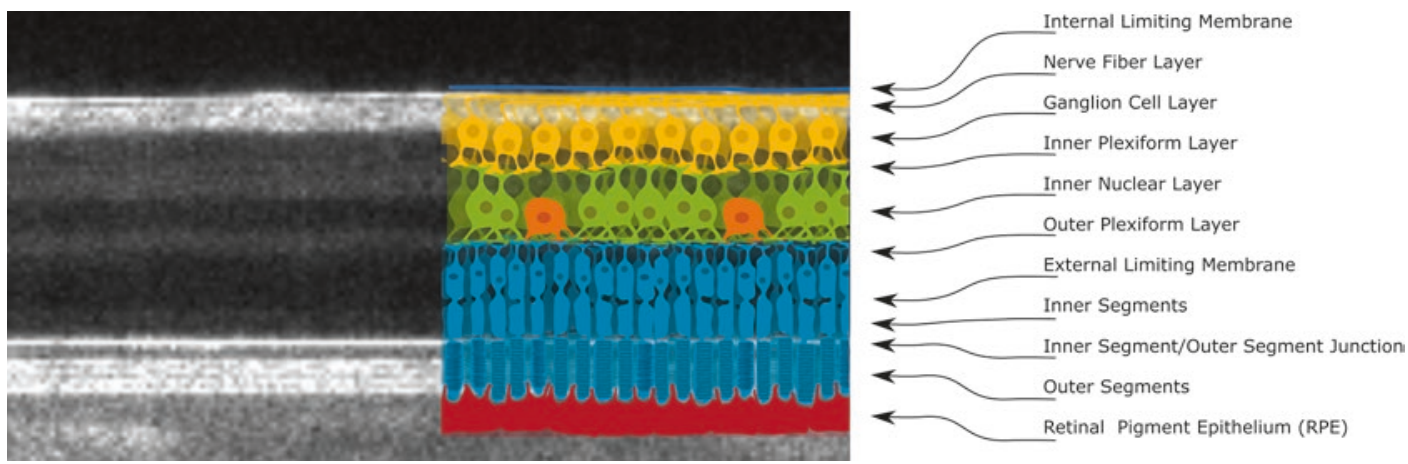

Fig. 8.1 Cross-sectional OCT image of human retina with the corresponding cellular structures

dimensional images that visualize anatomical structures and their pathological changes (Fig. 8.1) and is now indispensable for diagnosing a manifold of clinically important diseases [15-17]. OCT is based on interference [18] and detects not only the intensity of scattered light with an axial resolution of a few micrometers, but also optical path length changes, which are calculated from the phase of the interference pattern that generates the OCT signal (Fig. 8.2a). The phase carries information on morphological alterations in the nanometer range or percentage changes of the index of refraction. In addition, the phase information can also be used for numerical correction of aberrations [19] allowing imaging of the retina with single cell resolution. Combining aberration correction with interferometric measurements allows OCT to image quantitatively minute retinal changes.

Unfortunately, in retinal imaging the phase is usually dominated by ocular movements [20, 21]. These include drift, tremor, and microsaccades $[22,23]$. They are an integral part of the vision process and cannot be avoided completely. Microsaccades are sporadic, very fast movements, which reach amplitudes of up to $300 \mu \mathrm{m}$ and a speed of $10 \mathrm{~mm} / \mathrm{s}$. They occur every few seconds but can be suppressed for some time. Between the microsaccades the retina drifts continuously at a speed of about $100 \mu \mathrm{m} / \mathrm{s}$. An additional tremor causes $10-100 \mathrm{~Hz}$ oscillations with amplitudes in the order of the lateral resolution. Finally, axial motion of about $6 \mu \mathrm{m}$ is caused by the pulsation of the choroidal vessels [24]. The influence of these ocular motions on the phase in the OCT images depends on the speed of OCT imaging and the sampling pattern of the voxels.

In fact, much effort was spent to obtain phasestable data, i.e., data with usable phase information, in order to show some of the advantages of phase-stable imaging. Today's clinical FD-OCT systems measure 100,000 A-scans/s and raster scan the tissue in both lateral directions (Fig. 8.2b). Full phase stability is established in each A-scan and over parts of a B-scan which is acquired in less than $10 \mathrm{~ms}$. These OCT systems still do not reach the required phase stability to detect nanometer morphological changes with the required precision.

Imaging without lateral phase noise is possible by full-field (FF) OCT. Instead of using a scanning beam to image an area of the retina, it combines a collimated illumination of the sample with detection by an area camera (Fig. 8.2c). Using the time domain principle, phase stable imaging of en face planes within the retina was demonstrated [25]. By translating the reference mirror, volumes of $1.5 \mathrm{~mm} \times 1.5 \mathrm{~mm} \times 1.4 \mathrm{~mm}$ were measured in $1.3 \mathrm{~s} \mathrm{[26].} \mathrm{However,} \mathrm{within} \mathrm{the}$ 5 ms needed to move from one axial plane to the next, the phase relation between the en face images is lost.

Only significantly faster imaging can provide full three-dimensional phase stability. Utilizing a wavelength swept light source for spectral detection of the interference, full-field swept-source (FF-SS) OCT [27] measures a complete retina volume (Fig. 8.2d) in less than $10 \mathrm{~ms}$ [28, 29]. In these volumes, retinal motion affects mostly the spectral phase of all voxels, which reduces resolution and sensitivity similar to an unbal- 
a

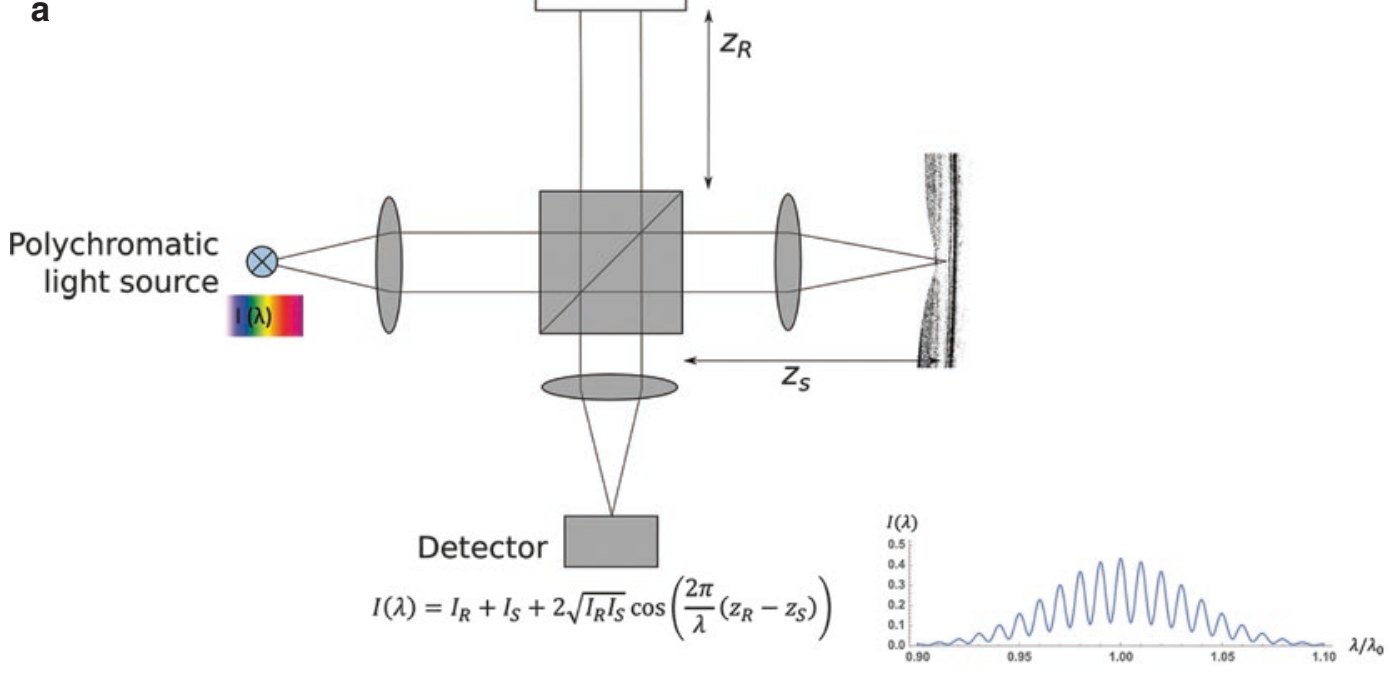

b

Scanning Fourier domain OCT

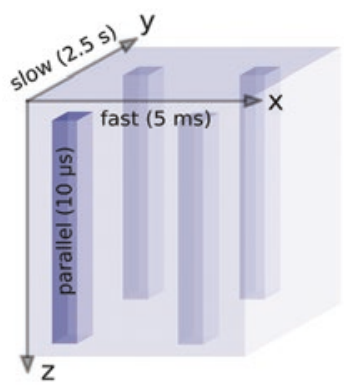

C

Full-field time domain OCT

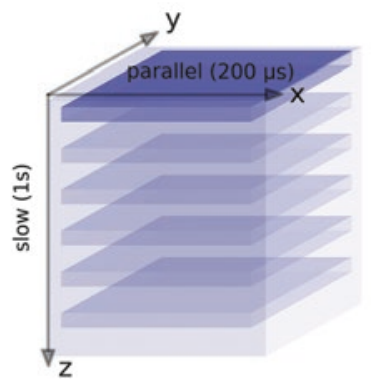

d

Full-field swept source OCT

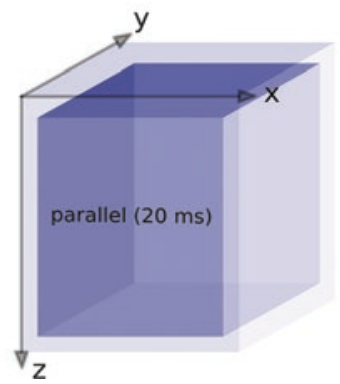

Fig. 8.2 Optical coherence tomography (OCT) for volumetric imaging of the retina. (a) Interference of polychromatic, broadband light is used to detect the distance of a scattering structure with high accuracy. Spectral intensity $I(\lambda)$ of the interference depends on the differences of the pathlengths $z_{R}$ und $z_{S}$ in reference and sample arm, respectively, and on the corresponding intensities $I_{R}$ and $I_{S}$. (b) Scanning Fourier domain (FD)-OCT imaging is currently used in clinical diagnosis. The axial information (amplitude and phase) is measured in parallel and the beam is raster scanned across the tissue to image the volume. (c) Full-field time domain (FF-TD) OCT measures en face

anced group velocity dispersion. This effect can be corrected by numerical post-processing [29, 30]. Hence, FF-SS-OCT gives access to the full three-dimensional phase information during in vivo retinal imaging [29].

Using the phase information, it becomes possible to refocus acquired images post factum [19, 31]. Even higher order aberrations can be cor- images in a few hundred microseconds. By scanning the en face plane, the full retina is acquired within seconds. The relative phase in each plane is not influenced by ocular motion, but the phase between the different planes is. (d) Full-field swept source (FF-SS) OCT images the full volume within $10 \mathrm{~ms}$. After correction of global axial motion, high resolution images of scattering amplitude and phase are reconstructed, which are free of motion artifacts. Volumetric imaging with minimal influence of eye motion on phase stability is achieved by parallel acquisition of lateral and axial information

rected, if the exact aberrations are known or if a method is conceived that yields them [31]. A system, such as FF-SS-OCT, that can obtain the entire phases, is, in a sense, a truly holographic OCT system-one that captures the whole information about the backscattered light field. We successfully developed such a system for retinal imaging to measure IOS. 


\subsubsection{Optical Setup}

The FF-SS-OCT setup used for imaging IOS of the retina (Fig. 8.3a) is described in detail elsewhere [31, 32]. In short, wavelength-swept light was generated by a tunable laser (Broadsweeper BS-840-1, Superlum, Ireland), which has a central wavelength of $840 \mathrm{~nm}$ and a bandwidth of $50 \mathrm{~nm}$. Its light was split by a fiber coupler into reference and sample arm. The sample light was collimated onto the area of interest on the retina, which then was imaged onto a high-speed camera (FASTCAM SA-Z, Photron, Japan). Superimposition with the collimated reference beam generates a hologram-like interference pattern, which was acquired with 60,000 frames per second. To get OCT volumes, 512 of those images were acquired during each wavelength sweep. Since the camera needs to store those images on internal memory, we were restricted to 70 volumes before the camera memory was exhausted.

For measurements of IOS, the retina was stimulated with a white light pattern. To this end, light of an LED, which was spatially modulated either by a simple mask or a modified projector, was coupled into the sample arm, such that a pattern was projected sharply onto the retina. A low-pass filter in front of the camera ensured that no light from the stimulation reached the camera. Synchronization of camera, swept laser, and projector was governed by an Arduino Uno microprocessor board. For measurements of IOS, healthy volunteers were positioned with a custom fit face mask in front of the setup to ensure repeatable measurement positions. The thermoplastic masks we used were originally designed for radiation therapy of the head [33] and proved to be a good alternative to a bite bar. After choosing an area of interest, fixation targets were selected and fixed in appropriate places. We then obtained OCT volumes at different areas with different stimulation intensities, stimulation frequencies, and time intervals between the stimulations. Written informed consent was obtained from all subjects. Compliance with the maximum permissible exposure (MPE) of the retina and all relevant safety rules was confirmed by a local safety officer. The study was approved by the ethics board of the University of Lübeck (ethics approval EthikKommission Lübeck 16-080).
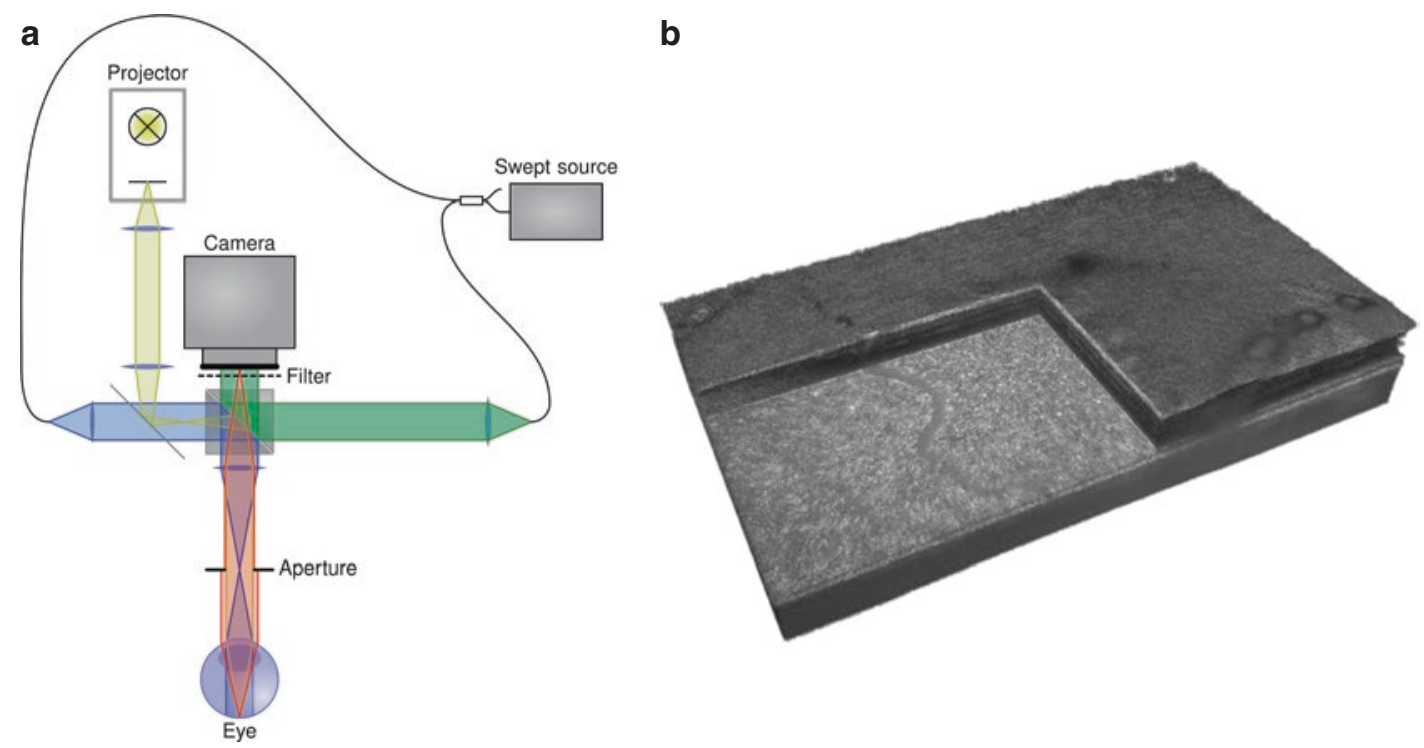

Fig. 8.3 (a) Full-field swept-source optical coherence tomography (FF-SS-OCT) setup for measuring intrinsic optical signals (IOS) of human retina. (b) Reconstructed volume of the retina 


\subsubsection{Data Evaluation}

FF-SS-OCT lacks a confocal gating. Therefore, it is highly vulnerable to multiple scattering and internal reflections within the setup. While multiple scattering is not severe in the neuronal retina, internal reflections show up as static horizontal lines and are hard to prevent. However, in contrast to any internal reflections, the retina itself is under constant movement. Averaging all 70 volumes in a dataset will therefore result in phase washout of the retinal structures, leaving only the static structures that are due to internal reflections. First step of the reconstruction is simply a subtraction of this averaged volume from each volume in the dataset.

The second step in the data evaluation is a standard Fourier domain OCT reconstruction. This is achieved by Fourier transforming all 512 images in each volume along the image number axis, i.e., the spectral axis, in order to retrieve the depth information. The Fourier transform of all 512 different wavelengths yields an A-scan for each pixel of the camera.

Since axial motion during the sweep changes the optical path length for each wavelength, it manifests in axial blurring that is essentially identical to group velocity dispersion (GVD) mismatch between reference and sample arm. We corrected this by multiplying the spectral data with an appropriate phase factor that compensates for the induced phase changes. Different methods can be used to obtain the correct phase factors [29-31]. In our scenario the phase factors were determined iteratively by optimizing axial resolution using an image-sharpness metric.

In OCT imaging ocular aberrations also manifest in phase factors. In contrast to the aforementioned phase factors, aberration related phase factors are applied to the two-dimensional lateral Fourier transform of each en face plane, instead of the axial Fourier transform of each A-scan. Thus, the aberrations were also corrected after the acquisition of the volumes by optimization of images sharpness [31]. To this end, wavefront phase errors were represented by a linear combination of Zernike polynomials and this combina- tion was varied in an optimization using a simplex-downhill algorithm and a gradient descent algorithm until the chosen sharpness metric, here the Shannon entropy, was minimized.

As final pre-processing step, prior to the actual phase evaluation, the layers and pixels that actually carry the information about the outer segment length need to be extracted. This can be achieved by various methods for co-registration of the different volumes and segmentation of the inner segment/outer segment junction (IS/OS). The outer segment tips are then assumed to be in constant distance to the segmented IS/OS. With these layers having been segmented, we can finally move to the phase evaluation.

The phases in any layer do not carry information about expansion of the photoreceptors. For this reason, one can only evaluate phase changes when comparing phases between two distinct layers and between two different time points. Therefore, each pixel of each reconstructed volume was first referenced to the respective coregistered pixel in one specific volume that was acquired prior to the beginning of the optical stimulus by subtracting the respective prestimulus phase from each phase value. This removes any spatial random dependence of the phase but leaves its temporal evolution untouched. Afterwards, the phases in the two layers of interest, i.e., the outer segment tips and the inner segment/outer segment junction, were extracted by complex averaging of the OCT signals of the segmented layers and their axially adjacent points. Finally, changes in the optical path length of the photoreceptor outer segments were obtained from the phase difference of the two layers [32].

The overall data evaluation after acquisition required multiple hours on standard PC hardware. A particular challenge is the robustness of segmentation and co-registration, in particular with respect to robustness among different retinas and different areas within the retina. Both, co-registration and segmentation, are further aggravated by the low signal-to-noise ratio of the FF-SS-OCT data and its increased imaging artifacts. 


\subsection{IOS of the Human Photoreceptor Cells}

During optical stimulation, time series were recorded with up to 70 volumes. After reconstruction we visualized single photoreceptors (Fig. 8.4a-c) fixed at their position in the field of view over several seconds. No changes in light scattering were observed after optical stimulation (Fig. 8.4a-c). However, calculating the phase difference between the inner segment/outer segment junction (IS/OS) and photoreceptor tips (Fig. 8.4d) showed a clear increase of the optical path length across the outer segments. This elongation is limited within the optical resolution of the eye to the area of stimulation (Fig. 8.4e). On the retina an image of the stimulating pattern is created by the phase difference evaluation. Aberration correction makes it possible to assign the IOS to single photoreceptor cells or rather identify photoreceptor cells which did not contribute to the IOS even though they were stimulated (arrows). During a repeated measurement, few minutes later, the specific cones that did not contribute to the IOS, again
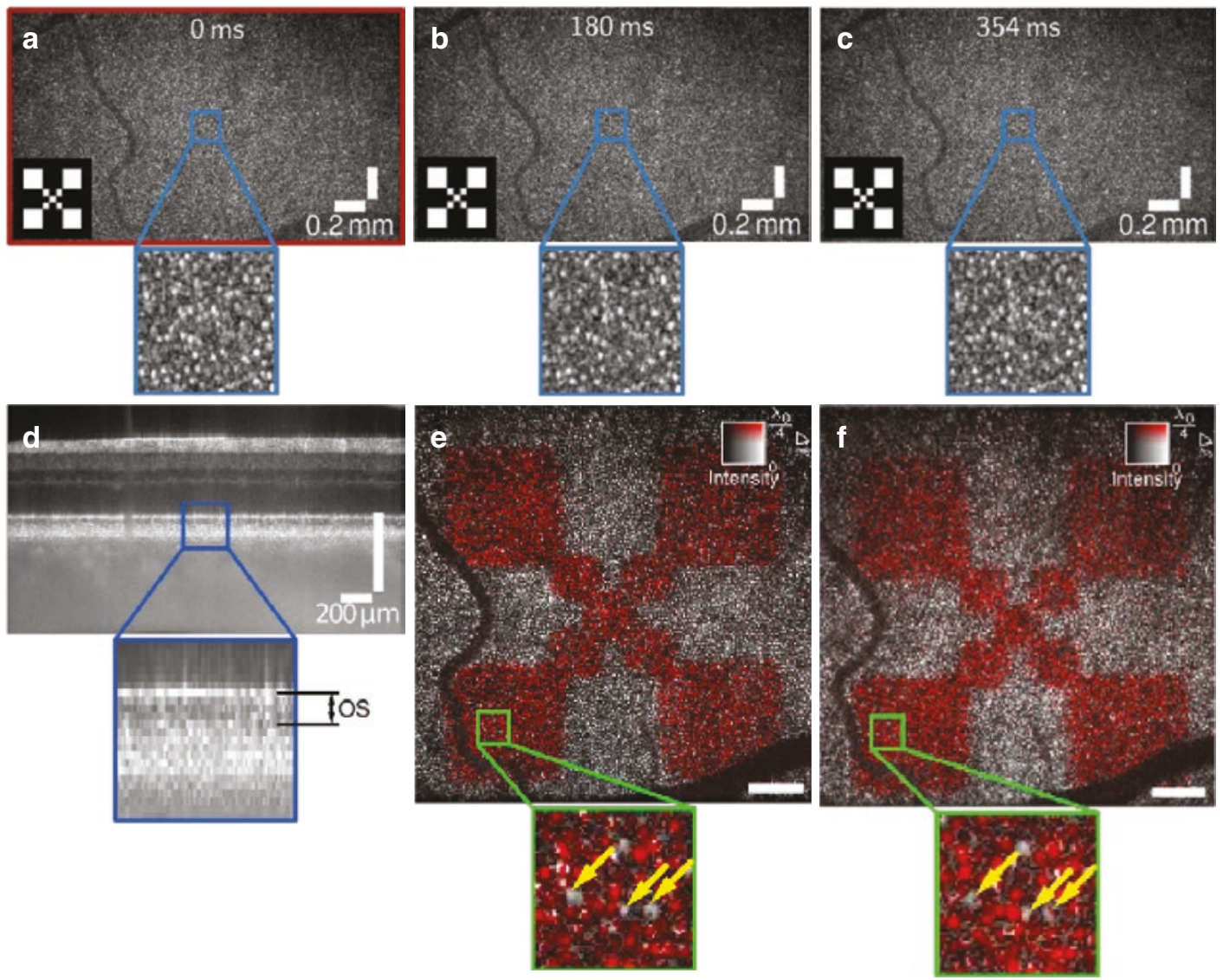

Fig. 8.4 Retinal imaging and response to an optical stimulus. (a-c) En face planes showing the backscattered intensity from the outer segment at the beginning of the stimulus, after $180 \mathrm{~ms}$, and after $384 \mathrm{~ms}$. Cone photoreceptors are clearly visible, but no change in the observed backscattering is detected. (d) Cross-sectional view (B-scan) from the center of the recorded volume. Changes of the optical path length within the outer segment were evaluated in this study. (e, f) Spatially resolved changes of the optical path length $\Delta l, 247 \mathrm{~ms}$ after switching off the stimulus pattern. A magnified view is shown in lower left inset. The measurement was repeated twice with a time interval of about $10 \mathrm{~min}$. Response is reproducible in the stimulated region. Noise was reduced by a lateral Gaussian filter. Scale bars are $200 \mu \mathrm{m}$. d-c reprinted with permission from Ref. [32] 
lack a response (Fig. 8.4f). However, it is currently not clear whether these photoreceptors truly do not react or whether the missing IOS is an imaging artifact.

For the evaluation of phase differences across the outer segments, resolving of single photoreceptors is not necessary. Even without large pupil diameters and/or aberration correction, clear phase signals were measured (Fig. 8.5a-d, g-j). For a short optical stimulus, the time course of the averaged phase differences was analyzed (Fig. 8.5k). First the optical path length in the outer segments shrinks by a few nanometers and then an elongation is observed, which continues long after the stimulation of the retina stopped. The time course of this elongation is highly reproducible in one person. However, the expansion rate differs from subject to subject although the general time course remains. The axial resolution of our FF-SS-OCT system is barely sufficient to discriminate the outer segment tips of rods and cones by their length. Still, different characteristic time courses with characteristic expansion rates were observed, when the phase signals from different depths was evaluated (Fig. 8.6a). The density of rods and cones depends on the retinal location. The macula contains mainly cones; the periphery is dominated by rods. The outer segment elongation in the periphery was faster than that one in the macula (Fig. 8.6b). This corroborates a different time course of the outer segment elongation of rods and cones.

Though we used different intensities of the stimulus, the initial expansion rate seems to be independent of both the duration and the intensity. Only the total duration of the expansion and, consequently, also the expansion amplitude changed. Evaluating the change of the optical path length in the outer segments, we obviously did not observe effects like bleaching or conformational changes of rhodopsin or neuronal activities, which are directly linked to the strength of the stimulus.

\subsubsection{Molecular Origin}

Using non-invasive optical measurements, retinal function has been investigated by different groups for quite some time $[8,9]$. In humans, first IOS were measured in photoreceptors using highspeed $(192 \mathrm{~Hz})$ retinal imaging with flood illumination and adaptive optics, which resolved single rods [14]. Clear changes in the intensity of backscattered light were observed for each cone upon optical stimulation. Later IOS were also observed with an adaptive optics SLO [11]. However, brightness of the reflected light increased or decreased randomly from cone to cone, which was explained by interference between light reflected from the IS/OS junction and the end tips of the cones. Both are highly scattering structures very well visible in OCT images (see Fig. 8.1). Depending on the length of the outer segments, an increase in the optical path length will increase or decrease the intensity of the interference depending on whether the reflected light interferes constructively or destructively, respectively. This ambiguity of the interference signal makes evaluation of the data difficult [34] and averaging over several cones cancels the signal. Hence resolving single photoreceptors by adaptive optics was essential. Using the interference internally in the outer segment, the observed signal is insensitive to axial motion but fails to give quantitative information about the morphological changes.

In contrast, OCT imaging gives direct information on phase changes in scattered light. The difference of the phase in the OCT signal at the IS/OS junction and the photoreceptor tips unequivocally determines path length changes unequivocally for every location on the retina. It is not necessary to resolve the photoreceptors. Averaging over larger areas with the same response is possible and increases the signal-tonoise ratio. Using FF-SS-OCT, we were able to measure these nanometer optical path length changes with millisecond resolution even in humans, despite the strong ocular motion. After a slight reduction of the path length during the stimulation, an increase up to half a micrometer was observed. With a typical length and index of refraction of the outer segments of $12 \mu \mathrm{m}$ and 1.41 , respectively $[35,36]$, this corresponds to $3 \%$ elongation. In mice the path length even increased up to $2 \mu \mathrm{m}$ or $10 \%$ under strong light stimulation [37]. Recent measurement tech- 
아앙

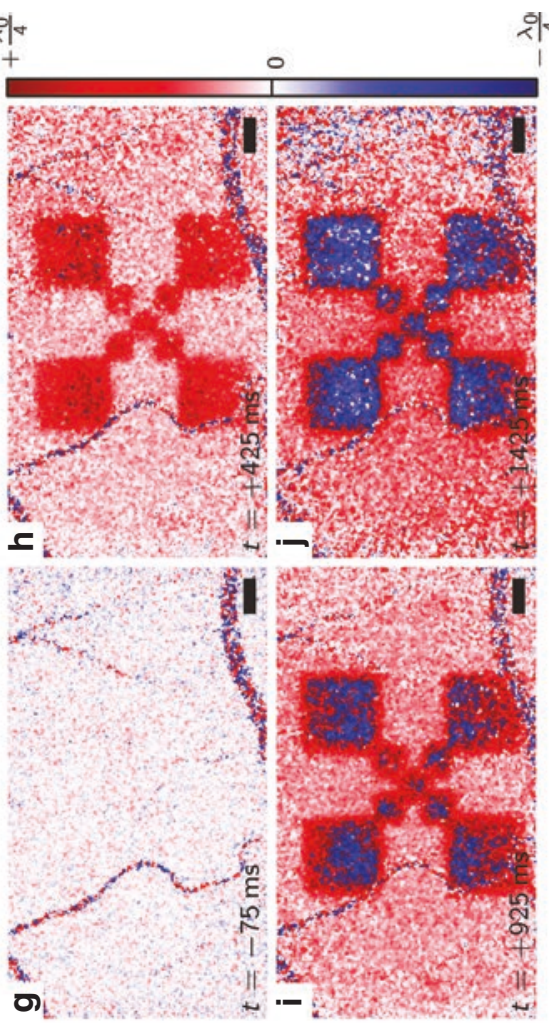

아만

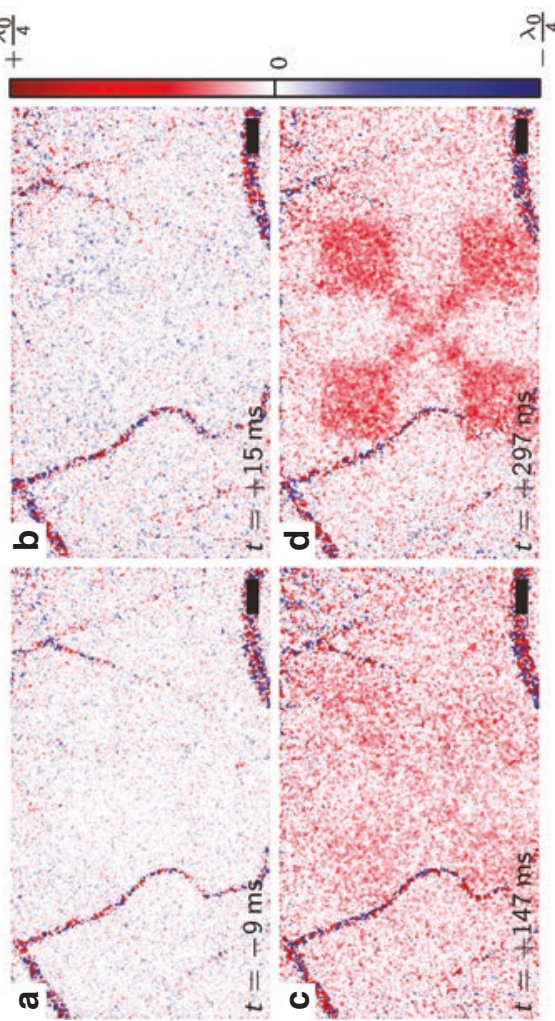

8
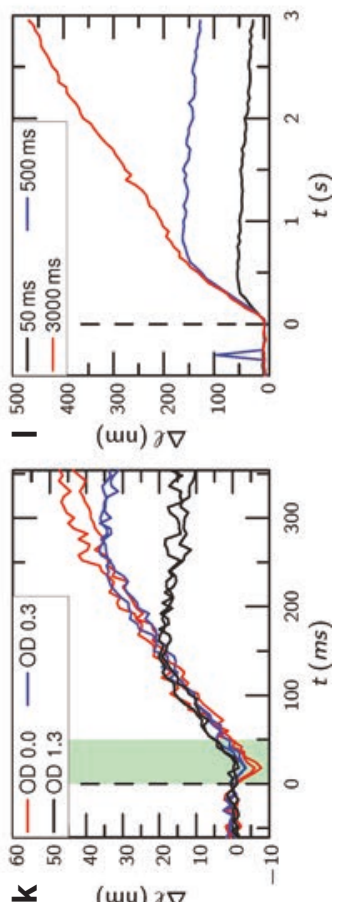

$+$
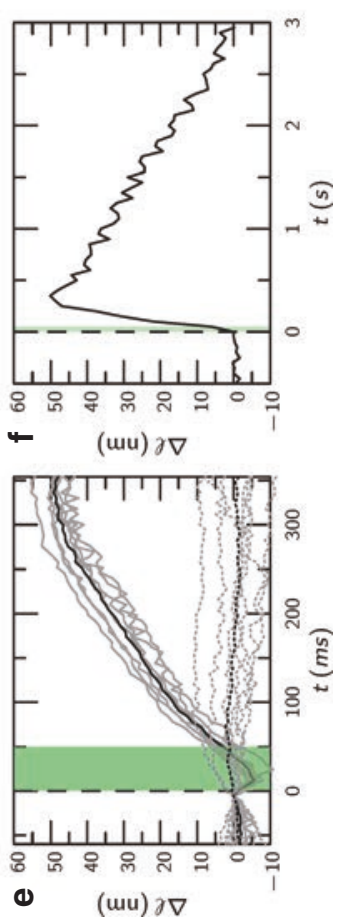

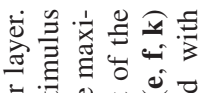

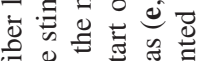

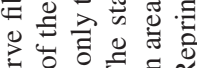

ये

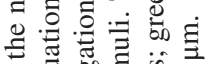

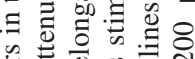

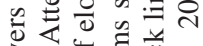

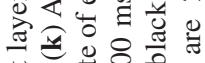

चี

屯ै

के

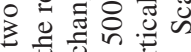

ปี

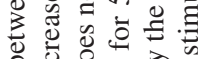

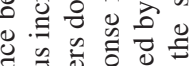

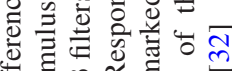

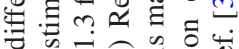

言

党

ข

0 웡

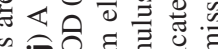

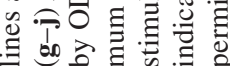

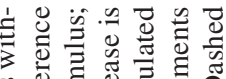

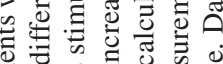

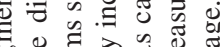

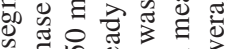

के

冚导

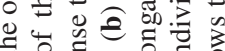

के

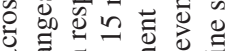

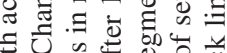

50.

ฮิ

돈

․

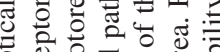

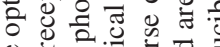

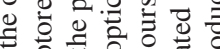

능을

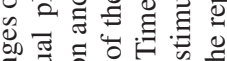

吅

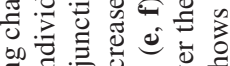

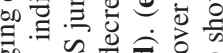

ฐ̊.

ก

$\infty \propto$

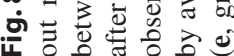


a

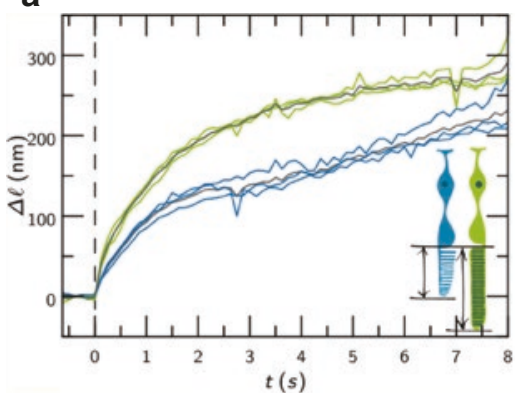

Fig. 8.6 Response of rods and cones to optical stimulation. (a) Evaluation of phase difference between IS/OS and different layers at the tips of the photoreceptors and the retinal pigment epithelium ( RPE) gives different time

niques using adaptive optics OCT with $\mathrm{MHz}$ A-scan rate, were also able reproduce the elongation of the outer segments in humans [38].

The observed changes of the optical path length are obviously caused by the biochemical processes within the outer segments. Here, the retina performs an extremely complex task converting photons into neuronal activity, which is eventually processed in the visual cortex. The vision process starts with the absorption of a photon by the molecule rhodopsin. The molecule is composed of the protein opsins which binds to the retinal molecule in 11-cis conformation. Absorption of light changes retinal to the alltrans conformation [39, 40]. Next, several biochemical amplification steps follow. The G protein transducin is activated by activated rhodopsin, by catalyzing an exchange of bound guanosine diphosphate (GDP) with guanosine triphosphate (GTP). This leads to the dissociation of a subunit of transducin, which then activates phosphodiesterase (PDE). Cyclic guanosine monophosphate (cGMP), which keeps $\mathrm{Na}^{+}$channels in the plasma membrane open, is hydrolyzed subsequently by the activated $\mathrm{PDE}$ to 5'-Guanosine monophosphate (GMP), the channels close, and the $\mathrm{Na}^{+}$concentration in the photoreceptor cell drops together with the voltage across the plasma membrane due to continuous active discharge of $\mathrm{Na}^{+}$from the photoreceptor cells. As result, the photoreceptors hyperpolarize and reduce their release of neu-

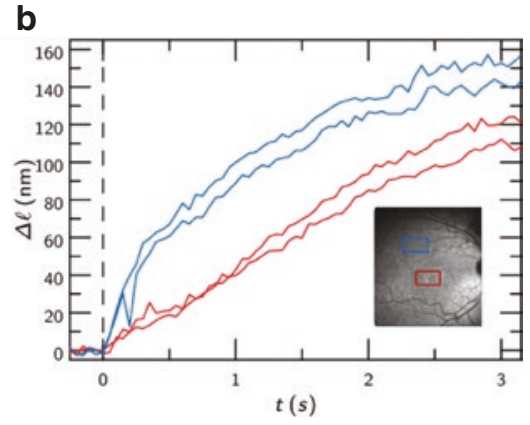

courses. (b) Kinetics of outer segment elongation depends on retinal location. In the periphery, which is dominated by rods, the optical path length increases faster than the macula, which contains only cones

rotransmitter, which either causes depolarization or hyperpolarization in the bipolar cells, depending on their subtype. Here and in a second inner neuronal layer of the retinal ganglion cells the visual information is processed before it is transmitted via non-myelinated axons to the brain. These axons form the nerve fiber layer and the optic nerve which leave the eye ball at the optic disk.

Although the observed elongation of the optical path length is clearly connected with the activity of the photoreceptor cells, it is unclear from which particular molecular process it originates. In the phototransduction cascade there are many opportunities for alteration of the optical path length. Change in absorption spectra of the photosensitive molecules or changes in the cellular concentration could lead to an alteration of the optical density and the refractive index. Generally, the index of refraction of solutions scales linearly with concentration of the solutes [36, 41]. However, under physiological conditions reasonable concentration changes would merely lead to a change of a few nanometers. For example, the index of refraction of phosphate buffered saline changes with only 0.01 per mol concentration change [42]. Concentration changes of $100 \mathrm{mmol}$ would change the optical path length by less than $10 \mathrm{~nm}$. This is much smaller than the observed elongation of the optical path length that reaches several hundred nanometers. Therefore, it seems reasonable that the increase of the optical path length is caused 
by a physical elongation of the outer segment, i.e., we observe conformational change of the outer segment by analyzing the phase of the OCT signal. Morphological changes of cells can be caused by different mechanisms. Cells change their shape actively with help of structural proteins like actin [43] or by changing the intracellular volume or the surface tension of the membranes [44]. The most likely explanation for the observed conformational change of the outer segment appears to be a compensation for osmotically driven volume change [37]. However, it is not completely clear which kind of concentration changes could lead to volume changes of several percent. The relocation of G-protein transducin was put forward as a driving mechanism for water influx which compensates the increase of osmotic pressure [37]. But also, active actinbased processes were observed in teleost rods, which lead to an elongation $[45,46]$.

\subsubsection{Technical Limitations of FF-SS-OCT}

To achieve holographic OCT three ingredients were key: First, we needed to acquire data as fast as possible in order to reduce eye motion during acquisition. Second, we needed to eliminate scanning artifacts by using FF-SS-OCT [28] and third, numerical motion correction, segmentation and registration had to be used to extract the phase information. Uniquely, holographic OCT imaging with more than $100 \mathrm{~Hz}$ volume rate and complete phase information was achieved, which no other imaging technology could provide so far. However, to allow imaging of the human retina with this technique, cameras with acquisition rates above $20 \mathrm{kHz}$ are required, $60 \mathrm{kHz}$ are preferable [29]. At the latter acquisition rate, we image the retina with up to $40 \mathrm{MHz}$ A-scan rate. An additional advantage of our full-field imaging technique is that the slowest axis, and thereby also the most vulnerable to phase errors, is the axial direction. In this axis phase errors due to global motion are identical in all A-scans and can be removed numerically $[29,30]$. The frame rate of $20-60 \mathrm{kHz}$ which is need for reasonable fields of view are currently only achieved by complex, bulky, and expensive high-speed CMOS cameras. For this reason, the otherwise simple and low-cost setup becomes currently highly expensive.

Besides many advantages, which are provided by FF-SS-OCT, there are also some disadvantages and restrictions. Image quality in each volume is comparably poor. This is caused by the fast imaging speed, which leads to a short integration time and therefore to a low number of detected photons per voxel. The low image quality combined with the huge amount of data which are generated by the fast imaging represents a serious challenge for the post-processing. For a phase sensitive evaluation of the measurements it is important to reference the phases to the exact same space, preferable with sub-pixel precision. For the detection of IOS in the retina, one major challenge in post processing is co-registration and segmentation of the region of interest. The huge data size and the high request in precision lead to long computation times accumulating to several hours.

The parallel imaging of the whole field of view precludes the suppression of multiply scattered light as confocal imaging does. As a consequence, the image quality suffers from crosstalk and is sensitive to reflections of the imaging optics including the cornea of the eye. Fortunately, the neuronal retina is not strongly scattering and ballistic photons by far dominate the imaging. However, imaging the choroid is severely degraded by multiple scattering from the retinal pigment epithelium (RPE).

\subsubsection{Outlook}

Phase sensitive measurements with FF-SS-OCT deliver unique information that strongly suggests clinical applications. So far, the diagnostics of diseases of the retina are mainly based on ERG or morphological changes seen in OCT imaging. With a functional analysis of the retina, an earlier diagnosis or a better therapy monitoring could 
become possible. Therapies could be adjusted, and unnecessary medication or treatments may be avoided. Furthermore, the retina is developmentally and anatomically the only sensory system, that is part of the central nervous system (CNS) [47]. Therefore, many neurodegenerative diseases of the CNS like Parkinson's disease [48, 49], Alzheimer's disease [50-54] and multiple sclerosis [55-57] correspond with morphological changes in the retina. It is reasonable to assume that functional changes precede those changes as well. Thus, the detection of IOS could expand from a clinical application in the retina to a clinical application of the whole CNS.

In the long run, phase sensitive detection of IOS can contribute to the basic research of neuronal behavior, wiring, and post-processing in the retina and thereby lead to a better understanding of vision and general conclusions about the behavior of the CNS. However, for a wellfounded research with clinical value it is necessary to unambiguously resolve the molecular origin of the IOS. Therefore, the next steps should be to resolve this question by further ex vivo and animal experiments, where different pathways of the molecular processes could be turned off and thereby excluded as possible molecular origin. For an investigation of neuron wiring it is necessary to visualize those changes in optical path length for other cell layers as well, for instance, for the inner nuclear layer, including the bipolar and amacrine cells, or the ganglion cell layer. The signals from those layers are expected to be weaker than the signal of the photoreceptor outer segments. Additionally, motion artifacts from the vessels, corrupting the IOS, become stronger in these layers. Provided cellular function causes here change in optical path length as well using sophisticated post-processing, changes in the optical path length should be visible in these depths, too.

\section{References}

1. Bruce KS, Harmening WM, Langston BR, Tuten WS, Roorda A, Sincich LC. Normal perceptual sensitivity arising from weakly reflective cone photoreceptors. Invest Ophthalmol Vis Sci. 2015;56(8):4431-8.
2. Harmening WM, Tuten WS, Roorda A, Sincich LC. Mapping the perceptual grain of the human retina. J Neurosci. 2014;34(16):5667-77.

3. Mendoza-Santiesteban CE, Fernandez-Cherkasova L, Echavarria OH, Rodriguez RC, Columbie-Garbey Y, Riesgo TJ. Multifocal electroretinography. Semin Ophthalmol. 2010;25(4):155-64.

4. Baker BJ, Kosmidis EK, Vucinic D, Falk CX, Cohen LB, Djurisic M, et al. Imaging brain activity with voltage- and calcium-sensitive dyes. Cell Mol Neurobiol. 2005;25(2):245-82.

5. Liu T, Liu X, Wen R, Lam BL, Jiao S. In vivo imaging rhodopsin distribution in the photoreceptors with nano-second pulsed scanning laser ophthalmoscopy. Quant Imaging Med Surg. 2015;5(1):63-8.

6. Sharma R, Schwarz C, Hunter JJ, Palczewska G, Palczewski K, Williams DR. Formation and clearance of all-trans-retinol in rods investigated in the living primate eye with two-photon ophthalmoscopy. Invest Ophthalmol Vis Sci. 2017;58(1):604-13.

7. Liebman PA, Jagger WS, Kaplan MW, Bargoot FG. Membrane structure changes in rod outer segments associated with rhodopsin bleaching. Nature. 1974;251(5470):31-6.

8. Yao X, Wang B. Intrinsic optical signal imaging of retinal physiology: a review. J Biomed Opt. 2015;20(9):090901.

9. Yao X, Son T, Kim TH, Lu Y. Functional optical coherence tomography of retinal photoreceptors. Exp Biol Med (Maywood). 2018;243(17-18):1256-64.

10. Harary HH, Brown JE, Pinto LH. Rapid light-induced changes in near infrared transmission of rods in Bufo marinus. Science. 1978;202(4372):1083-5.

11. Grieve K, Roorda A. Intrinsic signals from human cone photoreceptors. Invest Ophthalmol Vis Sci. 2008;49(2):713-9.

12. Srinivasan VJ, Chen Y, Duker JS, Fujimoto JG. In vivo functional imaging of intrinsic scattering changes in the human retina with high-speed ultrahigh resolution OCT. Opt Express. 2009;17(5):3861-77.

13. Teussink MM, Cense B, van Grinsven MJ, Klevering $\mathrm{BJ}$, Hoyng $\mathrm{CB}$, Theelen $\mathrm{T}$. Impact of motionassociated noise on intrinsic optical signal imaging in humans with optical coherence tomography. Biomed Opt Express. 2015;6(5):1632-47.

14. Jonnal RS, Rha J, Zhang Y, Cense B, Gao W, Miller DT. In vivo functional imaging of human cone photoreceptors. Opt Express. 2007;15(4):16141-60.

15. Adhi M, Duker JS. Optical coherence tomography-current and future applications. Curr Opin Ophthalmol. 2013;24(3):213-21.

16. Johannesen SK, Viken JN, Vergmann AS, Grauslund J. Optical coherence tomography angiography and microvascular changes in diabetic retinopathy: a systematic review. Acta Ophthalmol. 2018;97(1):7-14.

17. Ly A, Phu J, Katalinic P, Kalloniatis M. An evidencebased approach to the routine use of optical coherence tomography. Clin Exp Optom. 2019;102(3):242-59.

18. Fercher AF. Optical coherence tomography - development, principles, applications. Z Med Phys. 2010;20(4):251-76. 
19. Shemonski ND, South FA, Liu YZ, Adie SG, Scott Carney P, Boppart SA. Computational high-resolution optical imaging of the living human retina. Nat Photonics. 2015;9:440-3.

20. Shemonski ND, Adie SG, Liu YZ, South FA, Carney PS, Boppart SA. Stability in computed optical interferometric tomography (Part I): Stability requirements. Opt Express. 2014;22(16):19183-97.

21. Shemonski ND, Ahmad A, Adie SG, Liu YZ, South FA, Carney PS, et al. Stability in computed optical interferometric tomography (Part II): In vivo stability assessment. Opt Express. 2014;22(16):19314-26.

22. Sugita M, Zotter S, Pircher M, Makihira T, Saito K, Tomatsu N, et al. Motion artifact and speckle noise reduction in polarization sensitive optical coherence tomography by retinal tracking. Biomed Opt Express. 2013;5(1):106-22.

23. Sugita M. Polarization sensitive optical coherence tomography with retinal tracking and multiple frame processing. PhD thesis, Medical University of Vienna; 2014.

24. Spahr H, Hillmann D, Hain C, Pfäffle C, Sudkamp H, Franke $\mathrm{G}$, et al. [Imaging blood flow and pulsation of retinal vessels with full-field swept-source OCT]. Klin Monatsbl Augenheilkd. 2016;233(12):1324-1330.

25. Sudkamp H, Hillmann D, Koch P, Endt MV, Spahr $\mathrm{H}$, Münst M, et al. A simple approach for aberrationcorrected OCT imaging of the human retina. Opt Lett. 2018;43(17):4224-7.

26. Sudkamp H, Koch P, Spahr H, Hillmann D, Franke G, Münst M, Reinholz F, et al. In-vivo retinal imaging with off-axis full-field time-domain optical coherence tomography. Opt Lett. 2016;41(21):4987-90.

27. Povazay B, Unterhuber A, Hermann B, Sattmann H, Arthaber H, Drexler W. Full-field time-encoded frequency-domain optical coherence tomography. Opt Express. 2006;14(17):7661-9.

28. Bonin T, Franke G, Hagen-Eggert M, Koch P, Hüttmann G. In vivo Fourier-domain full-field OCT of the human retina with 1.5 million A-lines/s. Opt Lett. 2010;35(20):3432-4.

29. Pfäffle C, Spahr H, Hillmann D, Sudkamp H, Franke $\mathrm{G}$, Koch P, et al. Reduction of frame rate in fullfield swept-source optical coherence tomography by numerical motion correction. Biomed Opt Express. 2017;8(3):1499-511.

30. Hillmann D, Bonin T, Lührs C, Franke G, HagenEggert M, Koch P, et al. Common approach for compensation of axial motion artifacts in swept-source OCT and dispersion in Fourier-domain OCT. Opt Express. 2012;20(6):6761-76.

31. Hillmann D, Spahr H, Hain C, Sudkamp H, Franke G, Pfäffle C, et al. Aberration-free volumetric high-speed imaging of in vivo retina. Sci Rep. 2016;6:1-11.

32. Hillmann D, Spahr H, Pfäffle C, Sudkamp H, Franke G, Hüttmann G. In vivo optical imaging of physiological responses to photostimulation in human photoreceptors. Proc Natl Acad Sci U S A. 2016;117:13138-43.

33. Li G, Lovelock DM, Mechalakos J, Rao S, DellaBiancia C, Amols H, et al. Migration from full-head mask to "open-face" mask for immobilization of patients with head and neck cancer. J Appl Clin Med Phys. 2013;14(5):243-54.

34. Cooper RF, Tuten WS, Dubra A, Brainard DH, Morgan JIW. Non-invasive assessment of human cone photoreceptor function. Biomed Opt Express. 2017;8(11):5098-112.

35. Kraft TW, Schneeweis DM, Schnapf JL. Visual transduction in human rod photoreceptors. J Physiol. 1993;464(1):747-65.

36. Sidman RL. The structure and concentration of solids in photoreceptor cells studied by refractometry and interference microscopy. J Biophys Biochem Cytol. 1957;3(1):15-30.

37. Zhang P, Zawadzki RJ, Goswami M, Nguyen PT, Yarov-Yarovoy V, Burns ME, et al. In vivo optophysiology reveals that G-protein activation triggers osmotic swelling and increased light scattering of rod photoreceptors. Proc Natl Acad Sci U S A. 2017;114(14):E2937-46.

38. Azimipour M, Migacz JV, Zawadzki RJ, Werner JS, Jonnal RS. Functional retinal imaging using adaptive optics swept-source OCT at $1.6 \mathrm{MHz}$. bioRxiv. 2018;420240. https://doi.org/10.1101/420240.

39. Baylor D. How photons start vision. Proc Natl Acad Sci U S A. 1996;93(2):560-5.

40. Yau KW. Phototransduction mechanism in retinal rods and cones. The Friedenwald Lecture. Invest Ophthalmol Vis Sci. 1994;35(1):9.

41. Barber R, Ross KF, Tkaczyk S. Refractometry of living cells. Nature. 1953;171(4356):720-4.

42. Berlind T, Pribil GK, Thompson D, Woollam JA, Arwin H. Effects of ion concentration on refractive indices of fluids measured by the minimum deviation technique. Phys Status Solidi c. 2008;5(5):1249-52.

43. Pontes B, Ayala Y, Fonseca ACC, Romão LF, Amaral RF, Salgado LT, et al. Membrane elastic properties and cell function. PLoS One. 2013;8(7):1-13.

44. Ling T, Boyle KC, Goetz G, Zhou P, Quan Y, Alfonso FS, et al. Full-field interferometric imaging of propagating action potentials. Light Sci Appl. 2018;7:107.

45. Pagh-Roehl K, Wang E, Burnside B. Shortening of the calycal process actin cytoskeleton is correlated with myoid elongation in teleost rods. Exp Eye Res. 1992;55(5):735-46.

46. Liepe BA, Burnside B. Light-activation of teleost rod photoreceptor elongation. Exp Eye Res. 1993;57:117-25.

47. London A, Benhar I, Schartz M. The retina as a window to the brain-from eye research to CNS disorders. Nat Rev Neurol. 2012;9:44-53.

48. Devos D, Tir M, Maurage CA, Waucquier N, Defebvre L, Defoort-Dhellemmes S, et al. ERG and anatomical abnormalities suggesting retinopathy in dementia with Lewy bodies. Neurology. 2005;65(7):1107-10.

49. Archibald NK, Clarke MP, Mosimann UP, Burn DJ. The retina in Parkinson's disease. Brain. 2009;132(5):1128-45.

50. Danesh-Meyer HV, Birch H, Ku JYF, Carroll S, Gamble G. Reduction of optic nerve fibers in patients with Alzheimer disease identified by laser imaging. Neurology. 2006;67(10):1852-4. 
51. Blanks JC, Hinton DR, Sadun AA, Miller CA. Retinal ganglion cell degeneration in Alzheimer's disease. Brain Res. 1989;501(2):364-72.

52. Blanks JC, Torigoe Y, Hinton DR, Blanks RHI. Retinal pathology in Alzheimer's disease. I. Ganglion cell loss in foveal/parafoveal retina. Neurobiol Aging. 1996;17(3):377-84.

53. Blanks JC, Schmidt SY, Torigoe Y, Porrello KV, Hinton DR, Blanks RHI. Retinal pathology in Alzheimer's disease. II. Regional neuron loss and glial changes in GCL. Neurobiol Aging. 1996;17(3):385-95.

54. Parisi V, Restuccia R, Fattapposta F, Mina C, Bucci MG, Pierelli F. Morphological and functional retinal impairment in Alzheimer's disease patients. Clin Neurophysiol. 2001;112(10):1860-7.
55. Monteiro MLR, Fernandes DB, Apóstolos-Pereira SL, Callegaro D. Quantification of retinal neural loss in patients with neuromyelitis optica and multiple sclerosis with or without optic neuritis using Fourier-domain optical coherence tomography. Invest Ophthalmol Vis Sci. 2012;53(7):3959.

56. Green AJ, McQuaid S, Hauser SL, Allen IV, Lyness R. Ocular pathology in multiple sclerosis: retinal atrophy and inflammation irrespective of disease duration. Brain. 2010;133(6):1591-601.

57. Fisher JB, Jacobs DA, Markowitz CE, Galetta SL, Volpe NJ, Nano-Schiavi ML, et al. Relation of visual function to retinal nerve fiber layer thickness in multiple sclerosis. Ophthalmology. 2006;113(2):324-32.

Open Access This chapter is licensed under the terms of the Creative Commons Attribution 4.0 International License (http://creativecommons.org/licenses/by/4.0/), which permits use, sharing, adaptation, distribution and reproduction in any medium or format, as long as you give appropriate credit to the original author(s) and the source, provide a link to the Creative Commons license and indicate if changes were made.

The images or other third party material in this chapter are included in the chapter's Creative Commons license, unless indicated otherwise in a credit line to the material. If material is not included in the chapter's Creative Commons license and your intended use is not permitted by statutory regulation or exceeds the permitted use, you will need to obtain permission directly from the copyright holder. 